\title{
Stochastic Resonance in Neuronal Network Motifs with Ornstein-Uhlenbeck Colored Noise
}

\author{
Xuyang Lou \\ Key Laboratory of Advanced Process Control for Light Industry, Ministry of Education, Jiangnan University, Wuxi 214122, China \\ Correspondence should be addressed to Xuyang Lou; Louxy@126.com
}

Received 24 April 2014; Accepted 28 June 2014; Published 10 July 2014

Academic Editor: Ming Gao

Copyright (C) 2014 Xuyang Lou. This is an open access article distributed under the Creative Commons Attribution License, which permits unrestricted use, distribution, and reproduction in any medium, provided the original work is properly cited.

We consider here the effect of the Ornstein-Uhlenbeck colored noise on the stochastic resonance of the feed-forward-loop (FFL) network motif. The FFL motif is modeled through the FitzHugh-Nagumo neuron model as well as the chemical coupling. Our results show that the noise intensity and the correlation time of the noise process serve as the control parameters, which have great impacts on the stochastic dynamics of the FFL motif. We find that, with a proper choice of noise intensities and the correlation time of the noise process, the signal-to-noise ratio (SNR) can display more than one peak.

\section{Introduction}

Recently, the dynamics of networks of bioinspired neurons has received wide attentions in all branches of science. Stochastic resonance, as a nonlinear effect in which noise can enhance the detection of weak signals [1], is one of the central topics in theoretical and computational neuroscience. Kwon and Moon [2] investigated the role of different connectivity regimes on the coherence resonance of Hodgkin-Huxley neurons and found that spatial synchronization increases as characteristic path length shortens and firing frequency increases as clustering coefficient decreases. Ozer et al. [3] introduced a subthreshold periodic driving to a single neuron of the Newman-Watts small-world network consisting of biophysically realistic Hodgkin-Huxley neurons and found that the stochastic resonance phenomenon depends significantly also on the coupling strength among neurons and the driving frequency.

Noise can provide improvements in the representation of weak signals through stochastic resonance [4]. Gong et al. [5] analyzed the temporal coherence and the spatial synchronization of the stochastic Hodgkin-Huxley networks with channel noise and found that the random shortcuts can enhance the coherence and synchronization, which are absent in the regular network. It has been found that many studies have been carried out in an attempt to the role of noise in stochastic resonance of neural systems ranging from the microscale to the macroscale [6-9].

However, few reports are available on the stochastic resonance of the small-scale neural motifs [10] which are subnetworks occurring frequently in complex networks and believed to be basic building blocks of many networks. It is demonstrated that network motifs can perform specific functional roles and do exist in real biological networks, such as protein-protein interaction networks [11], brain functional networks [12], neuronal networks [13], and transcription regulatory networks [14]. Among the neural motifs, the feed-forward-loop (FFL) motif commonly occurs in dendrite and feed-forward structure, in which two neurons are unidirectionally coupled to the third one. Therefore, it is of practical importance to understand how the noise, especially the Ornstein-Uhlenbeck colored noise, contributes to the neuronal information processing in FFL motif.

The structure of the paper is as follows. First, we introduce the basic FitzHugh-Nagumo equation formulation of neuronal population dynamics in Section 2. The dynamical model is equipped with a noise current modeled by an Ornstein-Uhlenbeck $(\mathrm{OU})$ process. In Section 3, the effects of noise on the signal-to-noise ratio (SNR) are analyzed in the single neuronal population. We extend the model and explore 


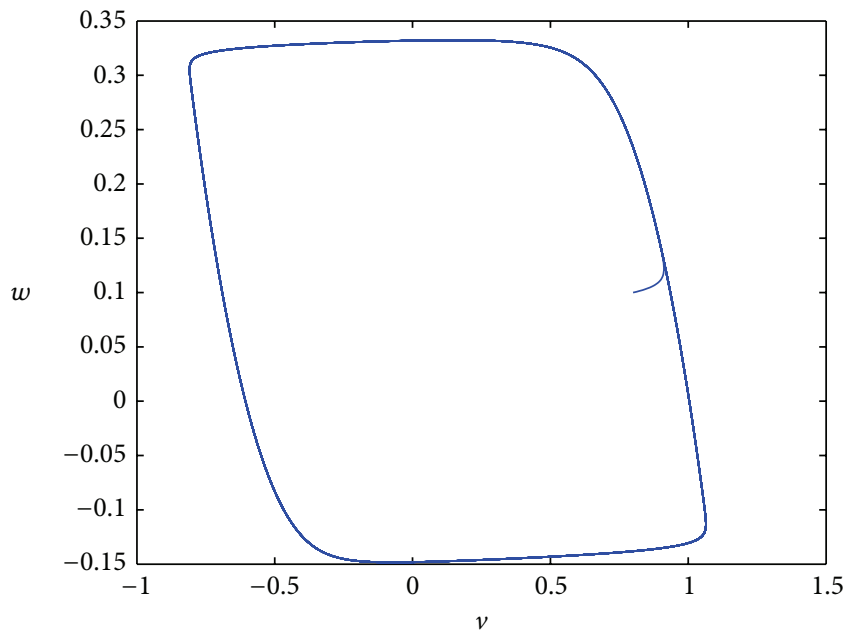

(a)

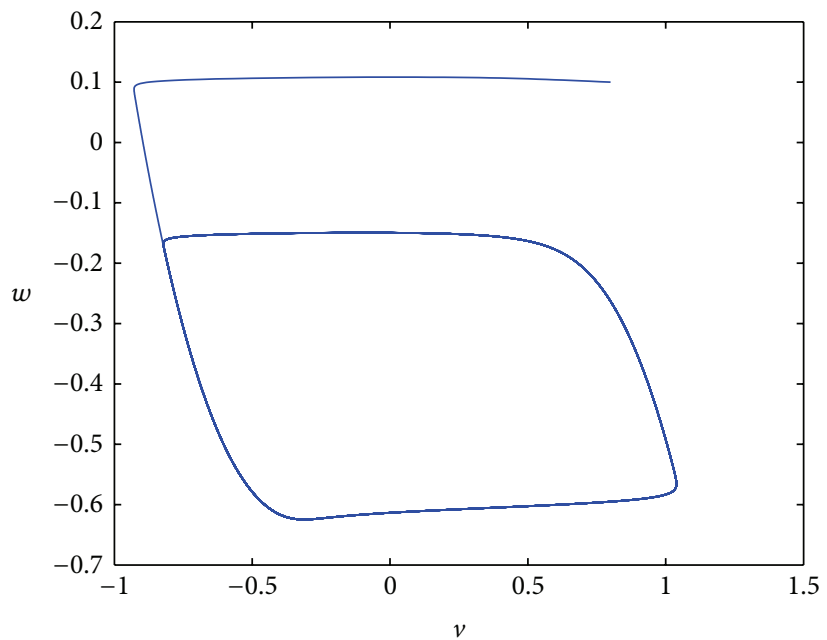

(b)

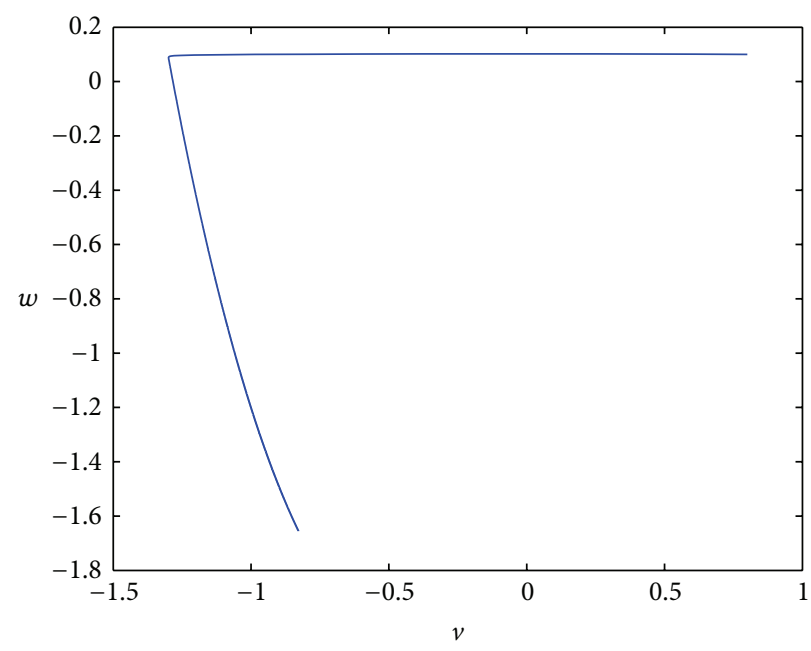

(c)

Figure 1: Phase plots of the FH model with initial values $v(0)=0.8$ and $w(0)=0.1$ and different current values: (a) $I=0.0$, (b) $I=-0.5$, and (c) $I=-2.0$.

the effects of colored noise on the SNR in the FFL neuronal motif in Section 4. Finally, a brief conclusion and discussion of our work are given.

\section{The Model}

The Hodgkin-Huxley model is of importance in describing the transmission of an action potential through a cell membrane [15]. However, due to the large number of variables, the phase space dynamics of the equation is hard to visualize. The FitzHugh-Nagumo (FH) neuron model has been proposed as a simplified model of the cell membrane [16], experimentally demonstrated by Nagumo et al. [17] using electrical circuits.

The FH model considered here is defined in a dimensionless form taken from [18]:

$$
\begin{gathered}
\epsilon \dot{v}=v(v+a)(1-v)-w+I, \\
\dot{w}=v-0.5 w,
\end{gathered}
$$

where $v$ is the voltage potential of the neuron membrane, $w$ is the inactivation of the sodium channels, and $I$ represents the input current. $\epsilon$ acts as the singular perturbation parameter and $w$ evolves on a much slower time scale than the voltage potential $v$. Here, we take the time scale separation variable $\epsilon=0.01$ and set the parameter $a=0.6$. For different current values, the neural system may exhibit complex dynamics (see Figure 1).

The total current input consists of the external applied current $I^{\mathrm{ext}}$, the total synaptic current $I^{\mathrm{syn}}$, and the noise current $\eta$; that is, $I=I^{\mathrm{ext}}+I^{\mathrm{syn}}+\eta$. In the single neuron level, the total synaptic current $I^{\text {syn }}$ will be taken as zero. For the noise current, the independent noise processes are governed by [19]$$
\dot{\eta}=-\frac{1}{\tau} \frac{\mathrm{d}}{\mathrm{d} \eta} U_{p}\left(\eta_{i}\right)+\frac{\sqrt{D}}{\tau} \xi(t)
$$ 


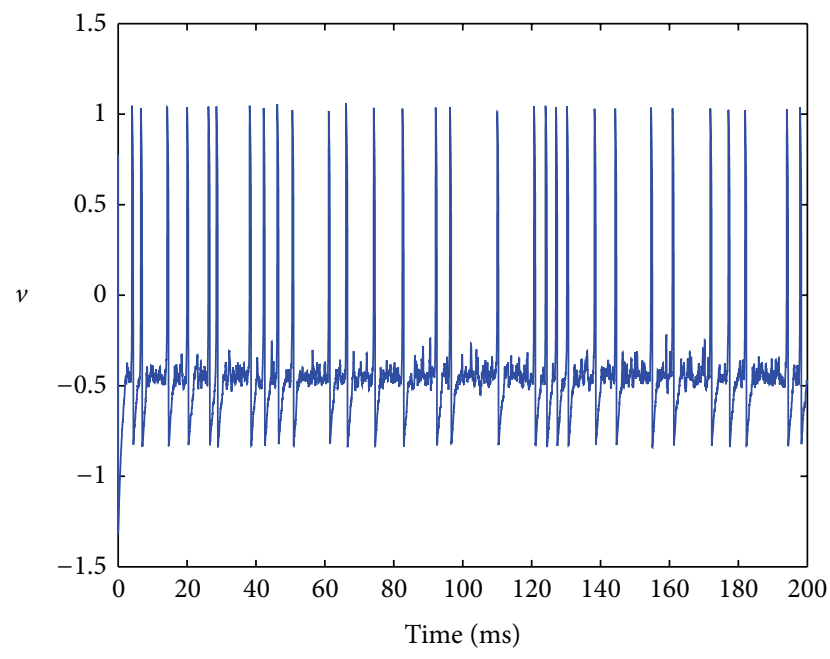

Figure 2: The time series of $v$ of the FH model with $D=6$ and $\tau=0.5$.

where the potential function is

$$
U_{p}(\eta)=\left(\frac{D}{\tau}(p-1)\right) \ln \left[1+\frac{\alpha(p-1) \eta^{2}}{2}\right]
$$

with $\alpha=\tau / D . \xi(t)$ is the Gaussian white noise process defined via $\left\langle\xi(t) \xi\left(t^{\prime}\right)\right\rangle=2 \delta\left(t-t^{\prime}\right)$ and $\langle\xi(t)\rangle=0 . D$ and $\tau$ define the intensity and the correlation time of the noise process. The form of the noise $\eta$ allows us to control the deviation from the Gaussian behavior by changing a single parameter $p$. For $p=1$, (2) becomes

$$
\dot{\eta}=-\frac{\eta}{\tau}+\frac{\sqrt{D}}{\tau} \xi(t)
$$

which is a well-known time evolution equation for the OU noise process [20].

\section{Single Neuron Level}

Before analyzing the effects of OU noise on the FFL neuron motif, let us now discuss the stochastic resonance in single neuron level. To do this, a localized weak rhythmic activity is introduced in the form of $I^{\mathrm{ext}}=I_{0}+A \sin (\omega t)$, which is added additively to the neuron. Here, $I_{0}=-2$ is the bias current and $A=0.01$ denotes the amplitude of the sinusoidal forcing current, whereas $\omega=0.5$ is the corresponding angular frequency. In this scenario, the external current is not large enough to excite the neuron without the induction of noise. We investigated the effects of noise as perturbation on the FH neuron in order to examine the stochastic oscillation phenomena. We set the noise intensity $D=6$ and the correlation time of the noise process $\tau=0.5$. As we see in Figure 2, regular spiking oscillations are induced due to the presence of the noise.

Next, we solved (1) and (4) numerically using a stochastic version of the Euler discretization scheme with a time step of $1 \mathrm{~ms}$ much smaller than the time constants of the problem.

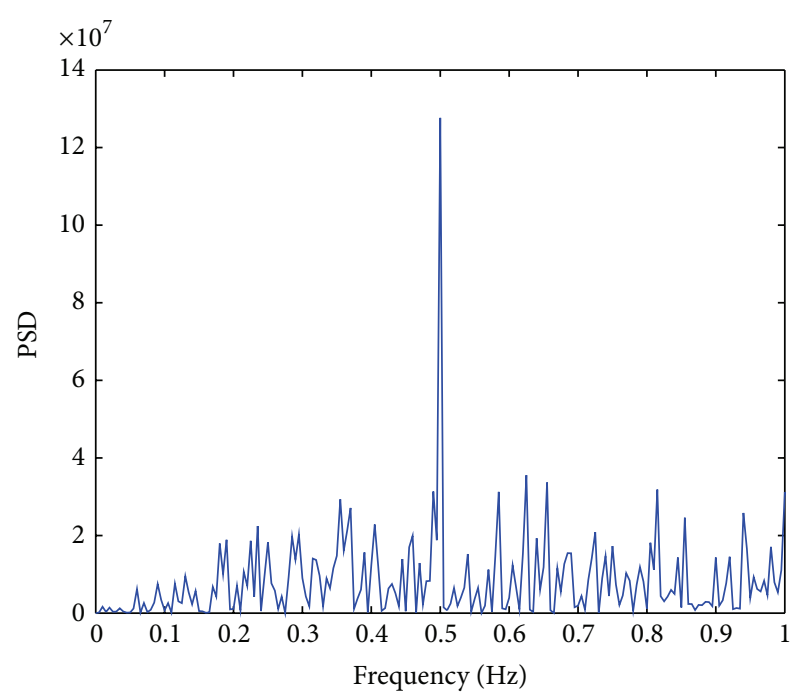

FIGURE 3: The power spectrum density graph of the time series of $v$ in Figure 2.

To quantitatively evaluate the performances of stochastic resonance, all data shown in our work refer to averages over 50 different realizations. Here, similar to [10], we use the SNR to measure the relative performance of stochastic resonance quantitatively. The SNR is one of the important measures for reducing the noise. There are different definitions for SNR. To calculate the SNR, the power spectral density (PSD) is first obtained from the time series of the membrane potential. An example of the PSD for the time series of $v$ in Figure 2 is depicted in Figure 3. It is seen that the PSD contains a main peak located at the forcing frequency $f=0.5 \mathrm{~Hz}$ and a background noise. This indicates that the frequency characteristic of the output spike train is induced by the local weak periodic forcing [10]. Define the SNR as $R=10 \log _{10}(S(f) / N(f))$, where $f$ is the input signal frequency, $S(f)$ denotes the value of the peak power at the frequency $f$ in the power spectrum of the time series of the output spike train, and $N(f)$ is the averaged power at nearby frequencies. A large SNR means that there is a larger variation of the signal amplitude than of the noise.

Now, we are ready to study the effects of different $D$ values on the relative performance of stochastic resonance. Figure 4 shows the SNR diagram with respect to the noise intensity as the control parameter. When $D$ is too small, there is almost no effect of noise on the spiking activities of the neuron. For an appropriate noise intensity, due to the excitatory effect of noise, the neuron starts to fire spikes. As a result of the increasing noise intensity, the SNR curves all first rise, then drop, and finally are maintained at a certain level, indicating that there exists an optimal noise intensity for the best performance.

\section{Motif Neuron Level}

Consider the FFL neuronal network motif based on coupled $\mathrm{FH}$ neurons given by

$$
\begin{gathered}
\epsilon \dot{v}_{i}=v_{i}\left(v_{i}+a\right)\left(1-v_{i}\right)-w_{i}+I_{i}, \\
\dot{w}_{i}=v_{i}-0.5 w_{i},
\end{gathered}
$$




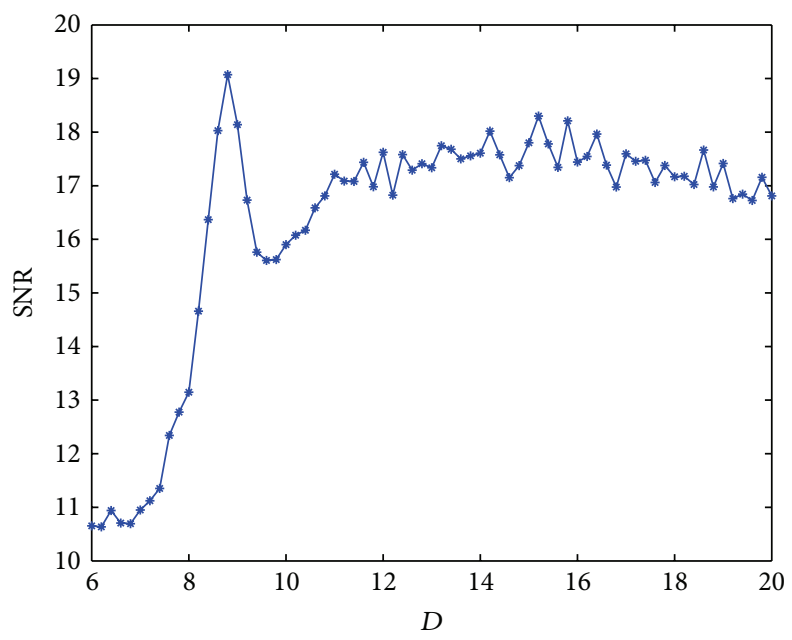

FIGURE 4: The SNR versus the noise intensity when $\tau=0.5$.

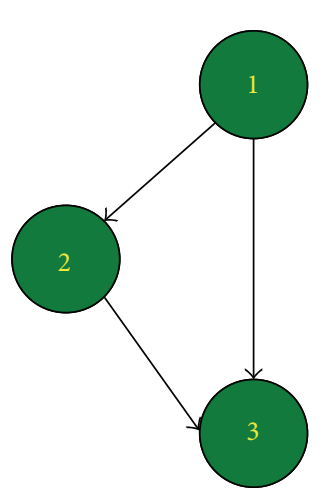

(a)

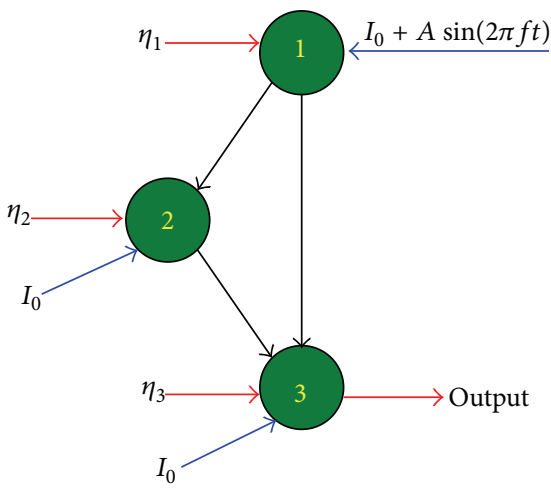

(b)

FIgURE 5: The FFL neuronal network motif. (a) The FFL motif. (b) Input-output structure of the FFL motif.

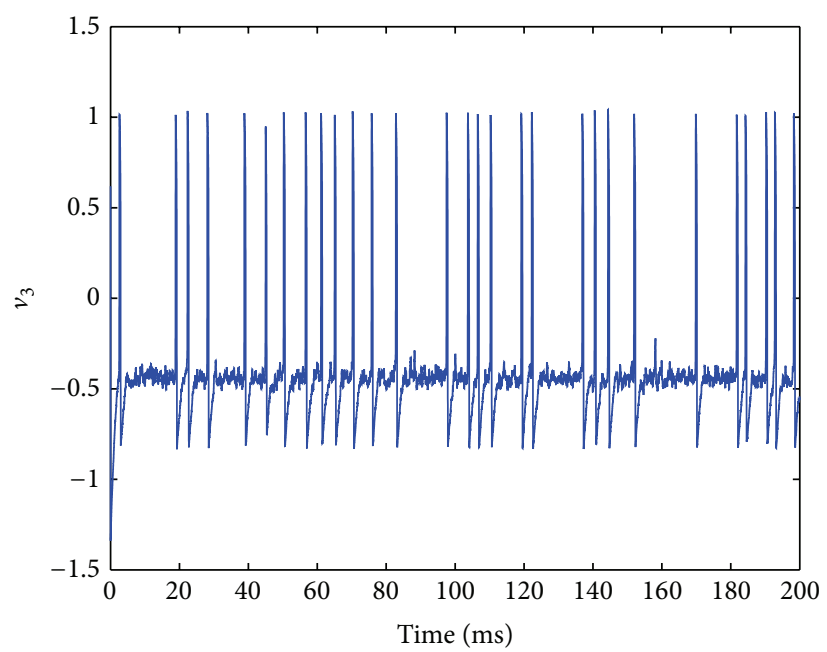

FIgURE 6: The stochastic oscillation of $v_{3}$ in the FFL motif, with $D=6, \tau=0.5$, and $g=0.113$. 


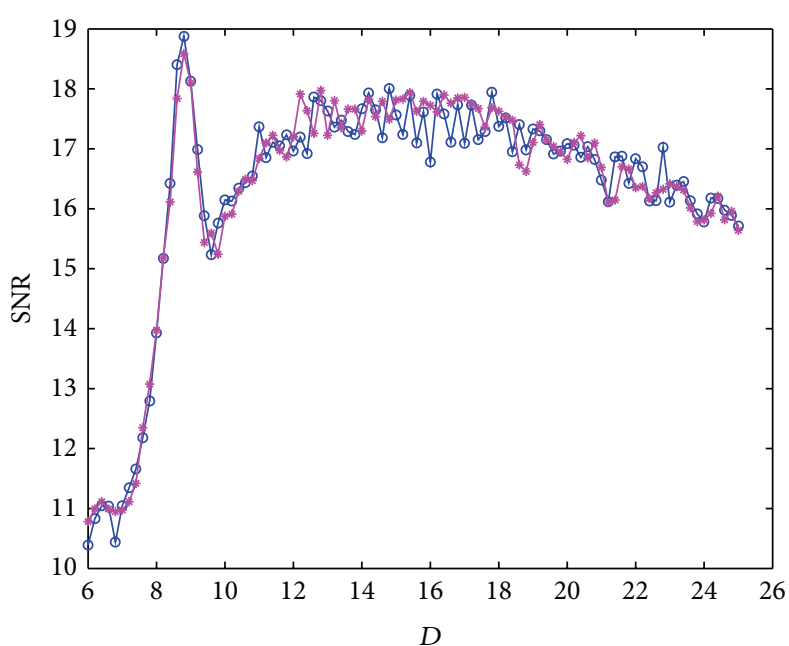

$\rightarrow g=0.1$

$\rightarrow-g=0.4$

(a)

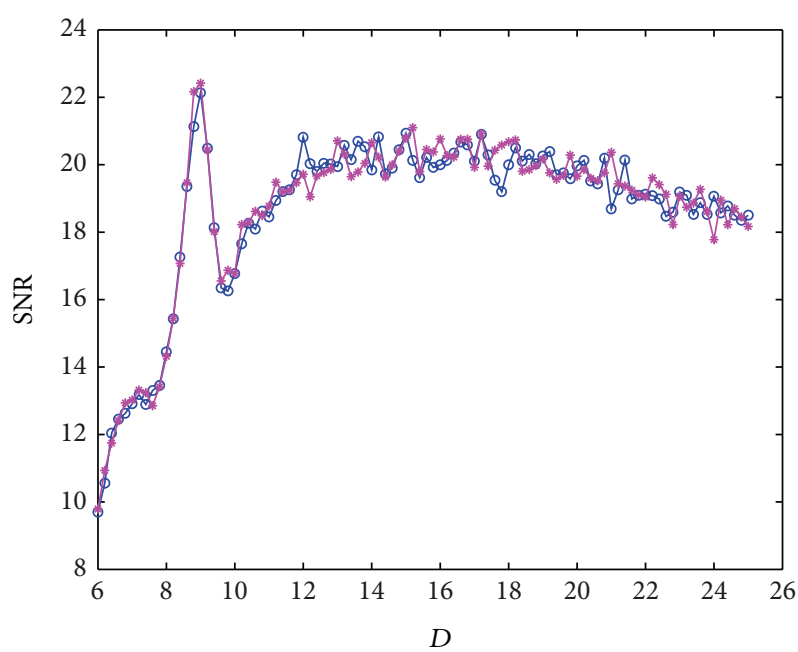

$\multimap g=0.1$

$\rightarrow-g=0.4$

(c)

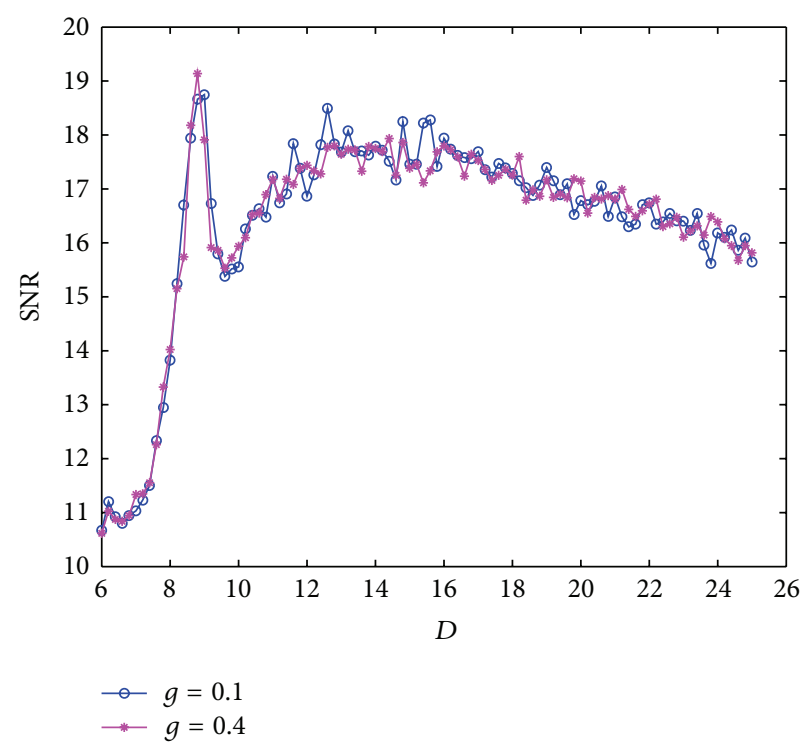

(b)

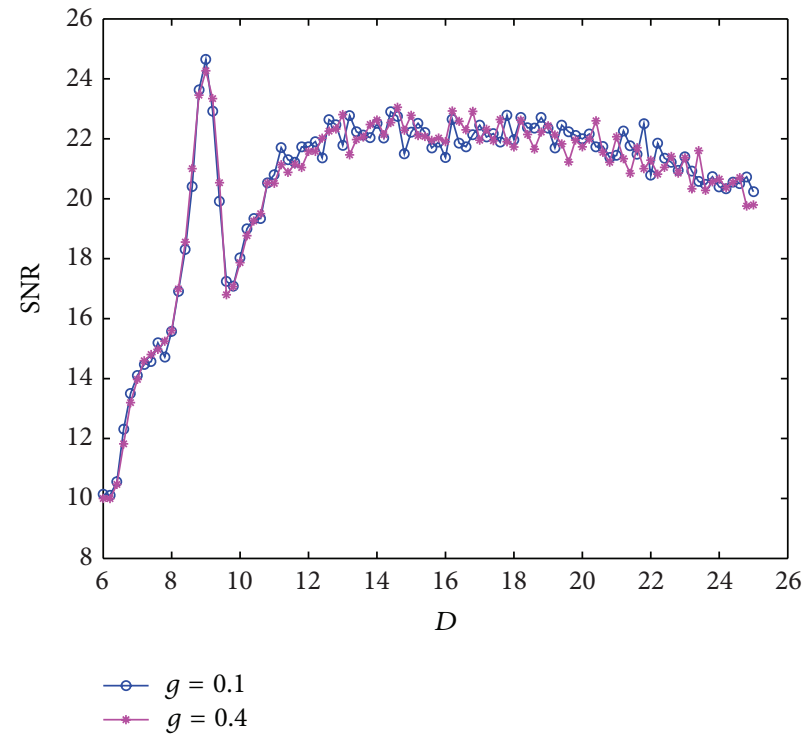

(d)

FIGURE 7: The SNR versus the noise intensity for different coupling strengths. (a) $\tau=0.2$, (b) $\tau=0.5$, (c) $\tau=0.8$, and (d) $\tau=1.1$.

where $i=1,2,3$ index the neurons, $v_{i}$ is the membrane potential of the $i$ th neuron, and $w_{i}$ is the inactivation of the sodium channels. The total current input $I_{i}$ consists of the external applied current $I_{i}^{\text {ext }}$, the total synaptic current $I_{i}^{\text {syn }}$, and the noise current $\eta_{i}$; that is, $I_{i}=I_{i}^{\text {ext }}+I_{i}^{\text {syn }}+\eta_{i}$. The external applied current $I_{i}^{\text {ext }}$ and the noise current $\eta_{i}$ are defined the same as in single neuron level. In this work, the synaptic current onto neuron $i$ will be the linear sum of the currents of all incoming synapses, $I_{i}^{\text {syn }}=-g \sum_{j=1}^{3} w_{i j} x_{j}(t)$, where $g$ describes the coupling strength of the synapse between neurons. $W=\left(w_{i j}\right)_{3 \times 3}$ is the Laplacian matrix of the network motif, where $w_{i j} \neq 0(i \neq j)$ implies that there is a connection from neuron $j$ to neuron $i . x_{i}$ represents membrane potential of neuron $i$. For simplicity, assume that the coupling strength is identical for all connections; that is, $g_{i j}=g$. For the noise current, the OU noise $\eta_{i}$ is given by

$$
\dot{\eta}_{i}=-\frac{\eta_{i}}{\tau}+\frac{\sqrt{D}}{\tau} \xi_{i}(t)
$$

In this section, we focus on the stochastic resonance of the coupled neurons in FFL motif structure. There are different configurations for FFL depending on the excitatory or inhibitory of the neurons in the motif. Here, we consider the FFL motif shown in Figure 5(a), that is, the T1-FFL type in [10], in which the first neuron and the second neuron are unidirectionally coupled to the third neuron.

In the following simulations, we use the same discretization scheme to solve (5) and (6) numerically. And the data 


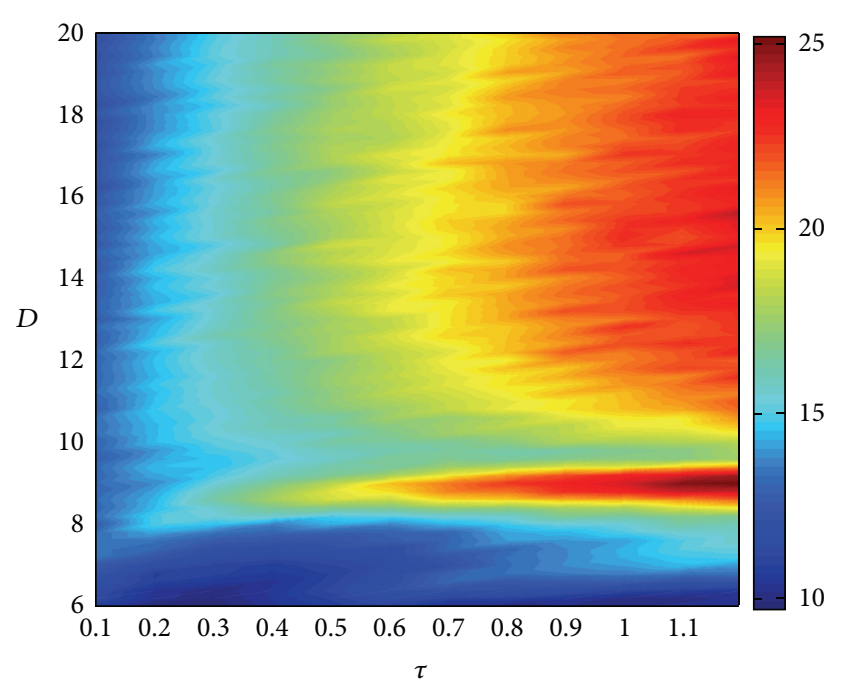

(a)

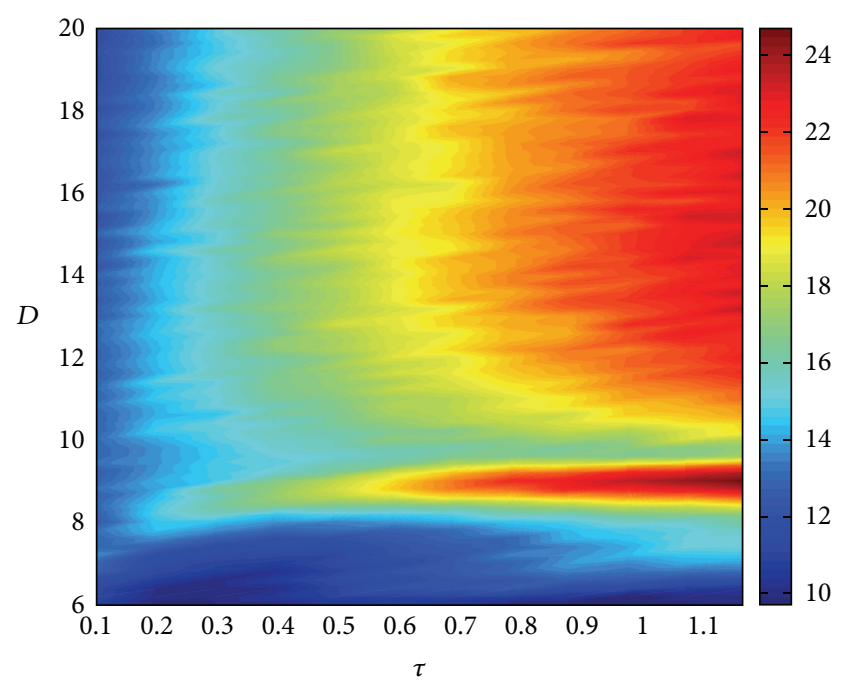

(b)

FIGURE 8: Color-featured SNR in dependence on $D$ and $\tau$ for different $g$. (a) $g=0.1$ and (b) $g=3.0$.

are averaged results of 50 independent runs in order to quantitatively evaluate the performances. As stated in [10], we only examine the response of neuron 3 to the external applied current of neuron 1 as neurons 1 and 3 are repetitively regarded as the input and output neurons of the FFL neuronal network motif. The input-output structure of the FFL motif is shown in Figure 5(b).

To examine whether the FFL neuronal network motif exhibits the stochastic resonance, we set $I_{2}^{\text {ext }}=I_{3}^{\text {ext }}=-2$, and a localized weak rhythmic activity is introduced in the form of $I_{1}^{\text {ext }}=I_{0}+A \sin (\omega t)$, which is added additively to the first neuron in (5). Here, $I_{0}=-2$ is the bias current and $A=$ 0.01 denotes the amplitude of the sinusoidal forcing current, whereas $\omega=0.5$ is the corresponding angular frequency. Different from the simulations in single neuron level, we will investigate the effects of the SNR not only on the strength of noise $D$ but also on the coupling strength $g$ and correlation time $\tau$ has been thoroughly studied. To examine the stochastic oscillation phenomena in the FFL motif level, we set the coupling strength $g=0.113$, the noise intensity $D=6$, and the correlation time of the noise process $\tau=0.5$. As we see in Figure 6 , the output $v_{3}$ of the FFL motif exhibits spiking oscillations induced by the noise in the first neuron.

In what follows, we will systemically analyze effects of different $D, \tau$, and $g$ on the relative performance of stochastic resonance via SNR. First, we examine the dependence of SNR on $D$ and $g$ with fixed values $\tau$. Figure 7 shows the SNR diagram with respect to the noise intensity as the control parameter. Similar phenomenon in Figure 4 can be observed from the curves. Due to the low input stimulus, there is almost no effect of noise on the spiking activities of the coupled neurons. However, along with the increasing noise intensity, the SNR starts to enhance and reaches a peak corresponding to an "optimal" noise intensity. In this case, the first neuron repeatedly fires spikes and then excitedly stimulates the other two neurons. Thus, the firing behaviors of these neurons almost display tonic firing activities and no quiescent state emerges. While further increasing the noise intensity larger than the "optimal" noise intensity, the SNR decreases quickly, indicating that the performance becomes worse. When the noise intensity $D$ is around 10 , the SNR reaches a valley point and then raises gradually again by further increasing the noise intensity $D$. Intuitively, the maximal SNR should turn larger while increasing values of the coupling strength $g$. However, this is not the case for the FFL motif with OrnsteinUhlenbeck colored noise here. As we see, as $g$ increases from 0.1 up to 0.4 , no explicit differences happen for the maximal SNR and the SNR curves. We further observe that how the scope of noise intensity for the maximal SNR changes with $\tau$. Evidently, the scope of noise intensity for the maximal SNR enlarges with increasing the correlation time of the noise process $\tau$ (see Figures $7(\mathrm{a})-7(\mathrm{~d})$ ).

Second, to gain more insights into the dependence of the SNR on $D$ and $\tau$, we calculate the $R$ on $D$ and $\tau$ by two different coupling strengths $g$. Figure 8 features the resulting color-contour plots for increasing values of $g$ from top to bottom. It is evident that there exists an optimal area for $9.0<$ $D<9.6$ and $1.0<\tau=1.2$ where the SNR is maximal, indicating the existence of noise-induced stochastic resonance and the optimal outreach of the localized activity of the output of neuron 3 .

\section{Conclusions}

In summary, we have considered the stochastic resonance of the FFL neuronal network motif subject to non-Gaussian noise. The FFL neuron motif has been built through the FitzHugh-Nagumo neuron model and the directed synapse couplings. We have mainly focused on the influence of noise on the stochastic resonance. The dependence of the SNR on the strength of noise $D$, coupling strength $g$, and correlation time $\tau$ has been thoroughly studied. It has been observed that 
the FFL motif can obtain high values of SNR at optimal noise intensities and proper coupling strengths.

\section{Conflict of Interests}

The author declares that there is no conflict of interests regarding the publication of this paper.

\section{Acknowledgments}

This work was supported by the National Natural Science Foundation of China (61104155), the Fundamental Research Funds for the Central Universities (JUSRP51322B), and the 111 Project (B12018).

\section{References}

[1] G. Mato, "Stochastic resonance in neural systems: effect of temporal correlation in the spike trains," Physical Review E, vol. 58, no. 1 , pp. 876-880, 1998 .

[2] O. Kwon and H. T. Moon, "Coherence resonance in small-world networks of excitable cells," Physics Letters A: General, Atomic and Solid State Physics, vol. 298, no. 5-6, pp. 319-324, 2002.

[3] M. Ozer, M. Perc, and M. Uzuntarla, "Stochastic resonance on Newman-Watts networks of Hodgkin-Huxley neurons with local periodic driving," Physics Letters A, vol. 373, no. 10, pp. 964-968, 2009.

[4] J. E. Levin and J. P. Miller, "Broadband neural encoding in the cricket cercal sensory system enhanced by stochastic resonance," Nature, vol. 380, no. 6570, pp. 165-168, 1996.

[5] Y. Gong, M. Wang, Z. Hou, and H. Xin, "Optimal spike coherence and synchronization on complex hodgkin-huxley neuron networks," ChemPhysChem, vol. 6, no. 6, pp. 1042-1047, 2005.

[6] T. P. Vogels and L. F. Abbott, "Signal propagation and logic gating in networks of integrate-and-fire neurons," The Journal of Neuroscience, vol. 25, no. 46, pp. 10786-10795, 2005.

[7] S. Wang, W. Wang, and F. Liu, "Propagation of firing rate in a feed-forward neuronal Network," Physical Review Letters, vol. 96, no. 1, Article ID 018103, 2006.

[8] E. M. Izhikevich, Dynamical Systems in Neuroscience: the Geometry of Excitability And Bursting, MIT Press, Cambridge, Mass, USA, 2007.

[9] O. V. Popovych, S. Yanchuk, and P. A. Tass, "Delay- and coupling-induced firing patterns in oscillatory neural loops," Physical Review Letters, vol. 107, no. 22, Article ID 228102, 2011.

[10] D. Q. Guo and C. G. Li, "Stochastic and coherence resonance in feed-forward-loop neuronal network motifs," Physical Review E, vol. 79, no. 5, 2009.

[11] S. Wuchty, Z. Oltvai, and A. Barabási, "Evolutionary conservation of motif constituents in the yeast protein interaction network," Nature Genetics, vol. 35, no. 2, pp. 176-179, 2003.

[12] O. Sporns and R. Kötter, "Motifs in brain networks," PLoS Biology, vol. 2, no. 11, pp. 1910-1918, 2004.

[13] S. Song, P. J. Sjöström, M. Reigl, S. Nelson, and D. B. Chklovskii, "Highly nonrandom features of synaptic connectivity in local cortical circuits," PLoS Biology, vol. 3, no. 3, pp. 507-519, 2005.

[14] U. Alon, An Introduction to System Biology: Design Principles of Biological Circuits, Chapman \& Hall, London, UK, 2006.

[15] G. Reddy and C. Murthy, Coherence Resonance in the FitzHughNagumo System, University of California, Physics 210B:
Nonequilibrium Statistical Physics, Student Project, San Diego, Calif, USA, 2013.

[16] R. FitzHugh, "Impulses and physiological states in theoretical models of nerve membrane," Biophysical Journal, vol. 1, no. 6, pp. 445-466, 1961.

[17] J. Nagumo, S. Arimoto, and S. Yoshizawa, "An active pulse transmission line simulating nerve axon," Proceedings of the IRE, vol. 50, no. 10, pp. 2061-2070, 1962.

[18] J. Keener and J. Sneyd, Mathematical Physiology, Springer, New York, NY, USA, 1998.

[19] B. C. Bag, K. G. Petrosyan, and C. Hu, "Influence of noise on the synchronization of the stochastic Kuramoto model," Physical Review E, vol. 76, no. 5, Article ID 056210, 2007.

[20] B. C. Bag and C. K. Hu, "Escape through an unstable limit cycle: resonant activation," Physical Review E: Statistical, Nonlinear, and Soft Matter Physics, vol. 73, no. 6, Article ID 061107, 2006. 


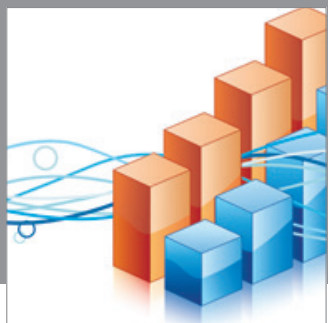

Advances in

Operations Research

mansans

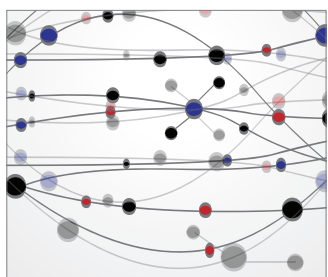

The Scientific World Journal
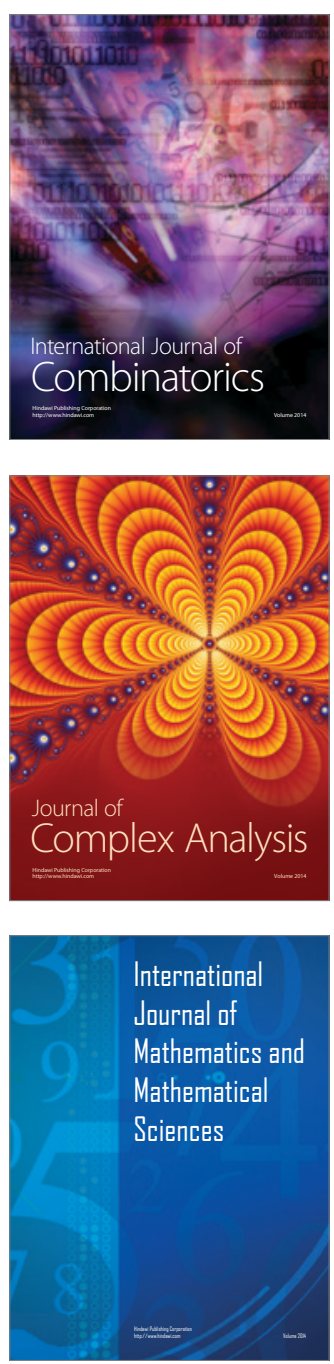
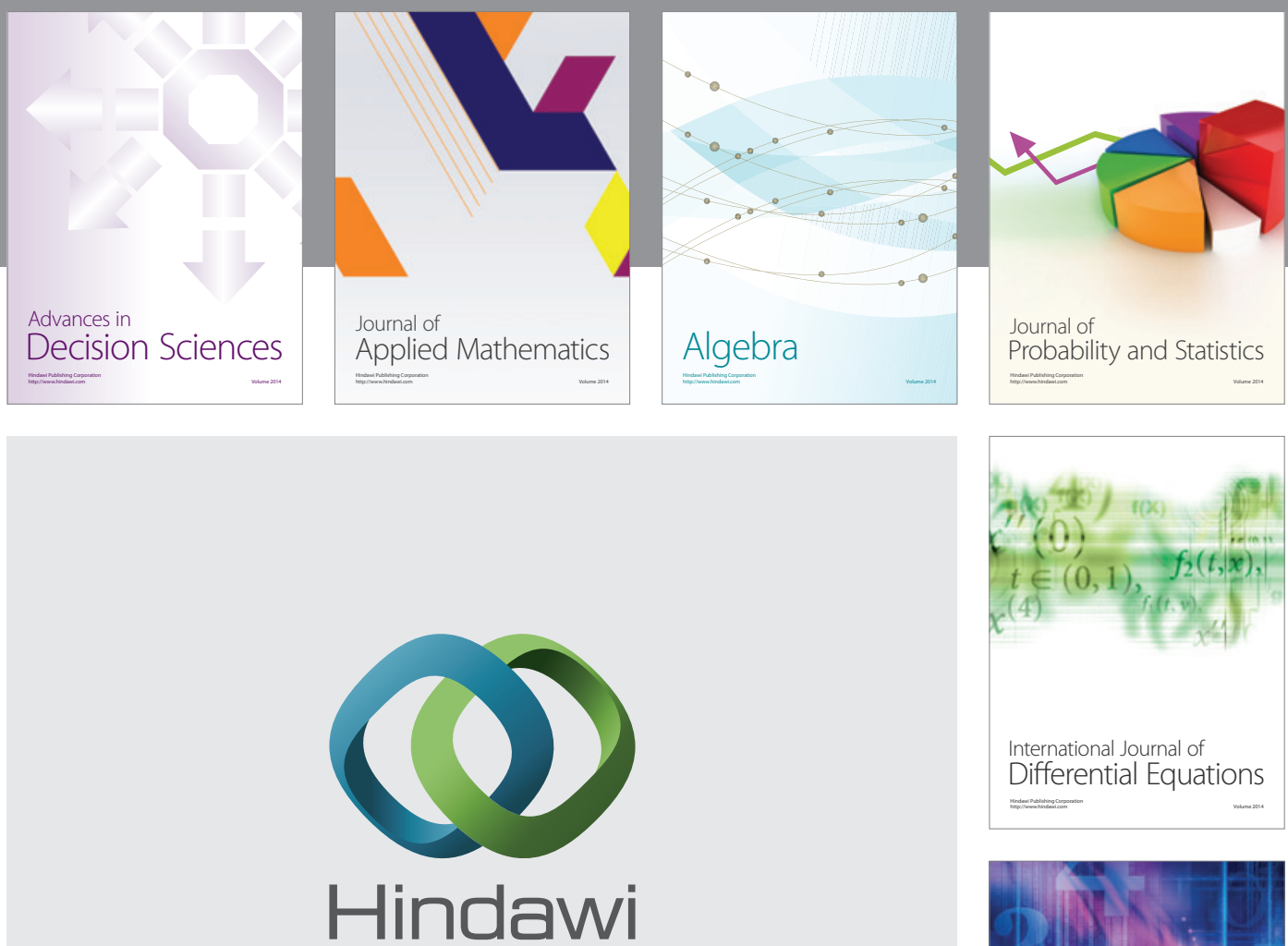

Submit your manuscripts at http://www.hindawi.com
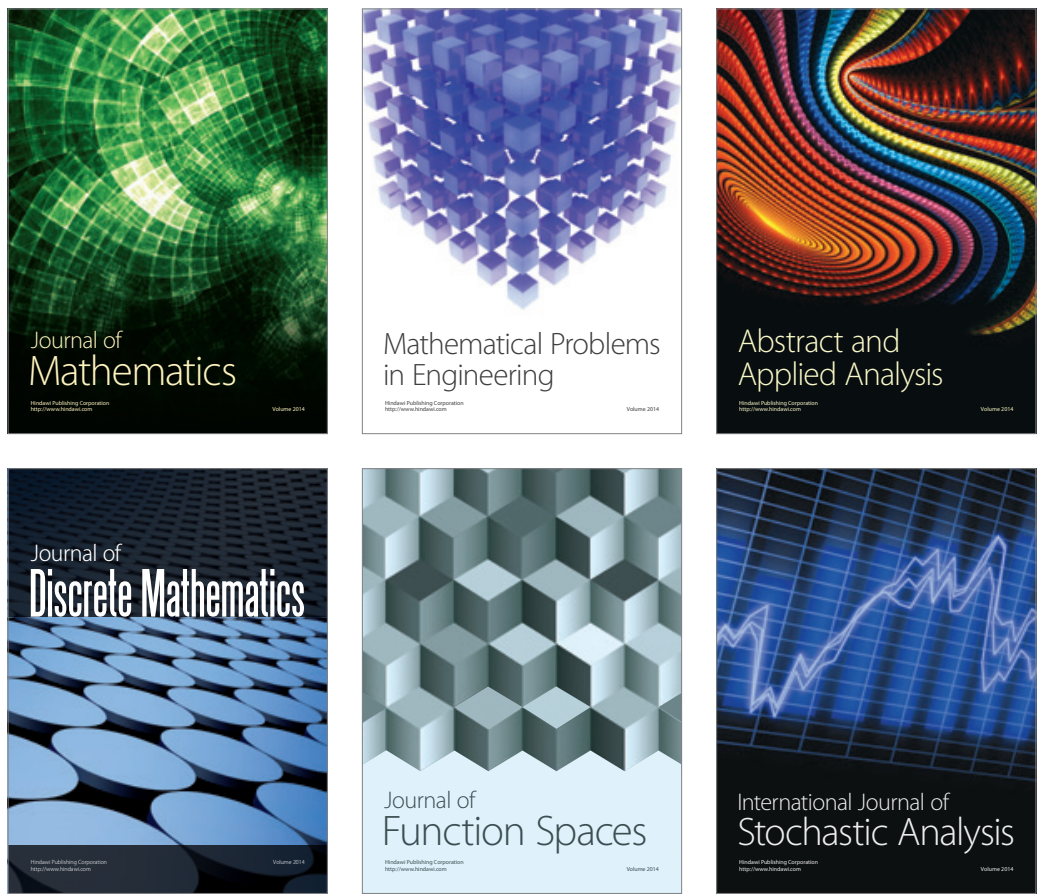

Journal of

Function Spaces

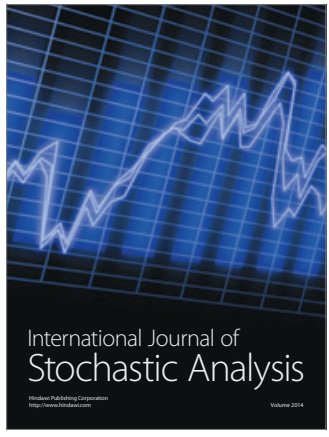

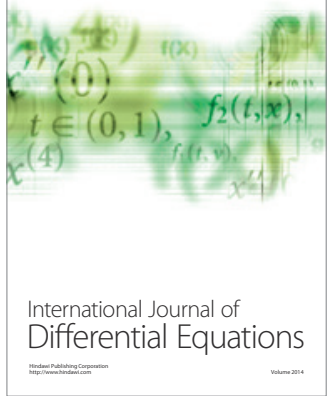
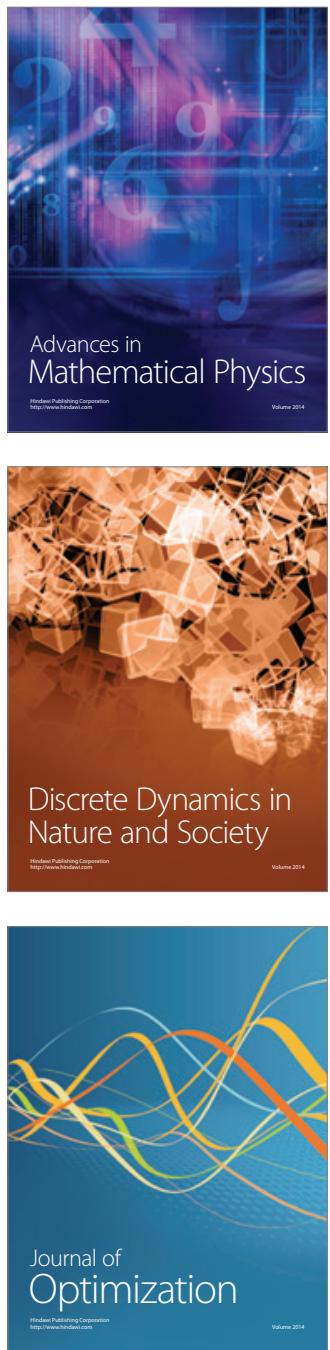